\title{
Índices de vegetação Modis aplicados na discriminação de áreas de soja
}

\author{
Joel Risso(1), Rodrigo Rizzi(2), Bernardo Friedrich Theodor Rudorff(1), Marcos Adami(1), \\ Yosio Edemir Shimabukuro(1), Antônio Roberto Formaggio(1) e Rui Dalla Valle Epiphanio(3)
}

\begin{abstract}
(1)Instituto Nacional de Pesquisas Espaciais, Caixa Postal 515, CEP 12245-970 São José dos Campos, SP. E-mail: risso@dsr.inpe.br, bernardo@dsr.inpe.br, adami@dsr.inpe.br, yosio@dsr.inpe.br, formag@dsr.inpe.br(2)Universidade Federal de Pelotas, Caixa Postal 354, CEP 96001-970 Capão do Leão, RS. E-mail: rodrigo.rizzi@ufpel.edu.br ${ }^{(3)}$ Noble Brasil S.A., Avenida das Nações Unidas, no 8.501, CEP 05425-070 São Paulo, SP. E-mail: ruiepiphanio@thisisnoble.com
\end{abstract}

Resumo - O objetivo deste trabalho foi avaliar o desempenho do índice de vegetação realçado (EVI) e do índice de vegetação da diferença normalizada (NDVI) - ambos do sensor "moderate resolution imaging spectroradiometer" (Modis) -, para discriminar áreas de soja das áreas de cana-de-açúcar, pastagem, cerrado e floresta, no Estado do Mato Grosso. Foram utilizadas imagens adquiridas em dois períodos: durante a entressafra e por ocasião do pleno desenvolvimento da cultura da soja. Para cada classe analisada, foram selecionadas 31 amostras de mapas de referência e avaliadas as diferenças nos valores de cada índice de vegetação, para a classe soja, foram avaliadas frente às demais classes, por meio do teste de Tukey-Kramer. Em seguida, foram avaliadas as diferenças entre os índices de vegetação, por meio do teste de Wilcoxon pareado. O NDVI apresentou melhor desempenho na discriminação das áreas de soja na entressafra, particularmente com uso das imagens do dia do ano (DA) 161 a 273, enquanto o EVI apresentou melhor desempenho no período de pleno desenvolvimento da cultura, especificamente com uso das imagens de DA 353 a 33. Portanto, o melhor resultado para classificação da soja, no Estado do Mato Grosso, via séries temporais do sensor Modis, pode ser obtida por meio do uso combinado do NDVI na entresssafra e do EVI no pleno desenvolvimento da soja.

Termos para indexação: classificação multitemporal de imagens, dados Modis, estimativas de área agrícola, imagens de satélite, sensoriamento remoto.

\section{Modis vegetation indices applied to soybean area discrimination}

\begin{abstract}
The objective of this work was to evaluate the performance of the enhanced vegetation index (EVI) and the normalized difference vegetation index (NDVI) - both from the moderate resolution imaging spectroradiometer (Modis) sensor - to discriminate soybean cultivated areas from sugarcane, pasture, cerrado, and forest ones in the state of Mato Grosso, Brazil. Images acquired during two periods were used: off-season and maximum soybean crop development. For each analyzed class, 31 samples were selected from reference maps, and the differences in the values of each soybean vegetation index were evaluated against the other classes using the Tukey-Kramer test. Afterwards, the differences between the vegetation indices were assessed using the Wilcoxon paired test. NDVI performed best in discriminating soybean areas during the off-season period, particularly when using images acquired from day of year (DOY) 161 to 273, whereas EVI performed best during maximum crop development, particularly when using images from DOY 353 to 33 . Therefore, best classification results for soybean in the state of Mato Grosso can be achieved by coupling Modis NDVI images acquired during off-season period and EVI images acquired during the maximum crop development period.
\end{abstract}

Index terms: multi-temporal image classification, Modis data, crop area estimates, satellite images, remote sensing.

\section{Introdução}

Monitorar sistemas dinâmicos, como a agricultura de ciclo anual, é um desafio que demanda recursos científicos e tecnológicos compatíveis com as escalas espaciais e temporais em que eles ocorrem. Nesse sentido, o programa "Earth observing system" (EOS) tem assumido papel categórico no desenvolvimento de satélites e de outras ferramentas para o estudo dos diversos processos que ocorrem nos sistemas terrestres. Um dos objetivos do programa é oferecer suporte para o monitoramento da agricultura e das florestas, com a disponibilização de imagens de sensores orbitais capazes de permitir inferências confiáveis sobre sua dinâmica espaço-temporal na superfície da Terra (Friedl et al., 2002; Justice et al., 2002).

Entre os sensores orbitais do programa EOS, destaca-se o sensor "moderate resolution imaging 
spectroradiometer" (Modis), lançado à bordo da plataforma Terra, em dezembro de 1999, e da plataforma Aqua, em maio de 2002 (Townshend \& Justice, 2002). O Modis combina algumas características do sensor "advanced very high resolution radiometer" (AVHRR) e dos sensores da série Landsat, com melhoria nas correções atmosféricas (Vermote et al., 2002) e geométricas dos dados (Wolfe et al., 2002), que são coletados em resolução temporal quase diária e resolução espacial de $250 \mathrm{~m}$ nas bandas do vermelho e do infravermelho próximo (Justice et al., 1998).

O EOS dispõe de aporte técnico-científico e estrutura organizacional eficiente para tratamento dos dados e posterior geração de produtos compostos, em diversos níveis (Townshend \& Justice, 2002). Entre eles, está o produto índices de vegetação, MOD13Q1 do Modis/ Terra, que é composto pelo índice de vegetação da diferença normalizada (NDVI) (Rouse et al., 1974) e pelo índice de vegetação realçado (EVI) (Huete et al., 1997). Ambos são gerados a partir do produto reflectância de superfície(MOD09) e resultam de observações efetuadas ao longo de intervalos regulares de 16 dias, em que os pixels de "melhor qualidade" são selecionados por meio da aplicação de algoritmo apresentado por Huete et al. (1999). A versão atual deste algoritmo é a 5.0, descrita em Didan \& Huete (2006).

De modo geral, o NDVIé mais sensível à presença de pigmentos que participam dos processos fotossintéticos, em particular ao conteúdo de clorofila (Gitelson et al., 1996; Huete et al., 1997, 2002), e minimiza algumas fontes externas de ruído como, por exemplo, sombras de nuvens (Justice et al., 1998). Essas propriedades, aliadas ao período decorrido desde a sua concepção, tornaram o NDVI um dos índices espectrais mais utilizados em estudos locais, regionais e globais (Zhang et al., 2003; Wardlow \& Egbert, 2008). No entanto, uma peculiaridade inerente ao NDVI é a saturação assintótica, o que o torna pouco sensível à detecção de variações de biomassa verde em condições de elevados índices de área foliar. O NDVI também é mais sensível a ruídos aditivos, tais como espalhamentos e absorções atmosféricos (Justice et al., 1998; Huete et al., 2002), e às condições do brilho de fundo do dossel (Huete, 1988).

O EVI foi desenvolvido para otimizar o sinal da vegetação, com melhoria na sensibilidade em condições de elevada biomassa, o que reduz as influências do sinal de fundo do dossel e da atmosfera (Justice et al., 1998). O EVI é mais sensível à estrutura do dossel (Huete et al.,
2002), embora sofra maior influência do ângulo zenital solar (Galvão et al., 2011) em determinadas condições. Essas melhorias são importantes para o monitoramento de culturas agrícolas por meio de índices de vegetação. No entanto, são escassos os trabalhos que exploram seu potencial para a identificação e o mapeamento de áreas de culturas agrícolas sobre extensas regiões (Wardlow et al., 2006).

Rizzi et al. (2009), por exemplo, propuseram o uso do "crop enhancement index" (CEI), que utiliza os valores de mínimo e máximo EVI das composições de imagens de 16 dias do Modis/Terra, dentro de um calendário agrícola bem definido, com vistas a identificar e mapear áreas de cultivo de soja. Ao ser aplicada no Estado do Mato Grosso, essa metodologia alcançou exatidão global superior a $91 \%$ na classificação das áreas de soja. O CEI foi adaptado e está sendo utilizado no Bioma Amazônia (Rudorff et al., 2012). Galford et al. (2008) e Arvor et al. (2011) avaliaram a expansão e a intensificação agrícola na região oeste do Mato Grosso, ao explorar diferentes peculiaridades das variações sazonais nas séries temporais do EVI do Modis. Wardlow et al. (2007) também estudaram as séries temporais dos índices de vegetação do Modis e demonstraram que o EVI e o NDVI apresentam potencial para discriminar diferentes culturas agrícolas em extensas áreas da Grande Planície Central dos Estados Unidos. Contudo, ainda são necessários estudos comparativos entre os dois índices de vegetação, para identificar características que permitam um melhor aproveitamento destes no âmbito do monitoramento agrícola.

O objetivo deste trabalho foi avaliar o desempenho dos índices EVI e NDVI, ambos do sensor Modis, para discriminar áreas de soja das áreas de cana-de-açúcar, pastagem, cerrado e floresta, no Estado do Mato Grosso.

\section{Material e Métodos}

A área de estudo compreende o Estado do Mato Grosso (Figura 1), localizado na porção sul da Amazônia Legal Brasileira $\left(7^{\circ} 10^{\prime} \mathrm{S}-18^{\circ} 0^{\prime} \mathrm{S} ; 50^{\circ} 0^{\prime} \mathrm{W}-\right.$ $\left.61^{\circ} 5^{\prime} \mathrm{W}\right)$, constituído por 141 municípios, que abrangem uma área de $903.358 \mathrm{~km}^{2}$. O estado é a principal fronteira agrícola brasileira (Galford et al., 2008) e foi responsável por $28 \%$ da produção nacional de soja na safra de 2011/2012 (Companhia Nacional de Abastecimento, 2012). 
Para a realização das análises, foram selecionadas, arbitrariamente, 31 amostras (pixels Modis puros), em cada uma das classes avaliadas: soja, cana-de-áçucar, pastagem, cerrado e floresta. Estas classes de uso/cobertura do solo foram escolhidas em razão da sua representatividade e, principalmente, pela disponibilidade de mapas de referência.

Para a classe soja, foi utilizado mapa de referência (Epiphanio et al., 2010) elaborado por meio da interpretação visual de imagens do sensor "thematic mapper" (TM)/Landsat 5, com o auxílio de dados obtidos em campo. As imagens utilizadas na elaboração deste mapa foram adquiridas no período de máximo desenvolvimento vegetativo da soja, na safra de 2005/2006, de acordo com o calendário agrícola do Estado do Mato Grosso (Epiphanio et al., 2010).

As amostras coletadas sobre as áreas de cana-de-açúcar foram selecionadas tendo-se utilizado, como referência, o mapa de cana-de-açúcar do projeto Canasat de 2006 (Rudorff et al., 2010), sobre áreas em produção.

A seleção das amostras coletadas sobre as áreas de cerrado foirealizada combasenomapa deremanescentes preservados do Bioma Cerrado, fornecido pelo
Ministério do Meio Ambiente e atualizado em 2008. Nesse mapa, constam apenas as classes de cerrado natural e as áreas sob ação antrópica, sem distinção dos tipos de uso do solo (Brasil, 2009).

A seleção das amostras de pastagem utilizou, como referência, o mapa do Projeto de Conservação e Utilização Sustentável da Diversidade Biológica Brasileira (Probio), de 2002, que, entre outras classes, inclui pastagem cultivada (Brasil, 2007). Para verificar se as áreas de pastagem não tiveram sua ocupação alterada até 2006, foi utilizada a ferramenta de visualização de séries temporais Modis (Freitas et al., 2011). A decisão de incluir cada amostra de pastagem na análise foi tomada com base no critério da não alteração do padrão do perfil temporal do índice de vegetação entre 2002 e 2006.

Os locais das amostras de floresta foram escolhidos com base no mapa das áreas remanescentes de floresta, do projeto Monitoramento da Floresta Amazônica Brasileira por Satélite, de acordo com o mapeamento realizado em 2007 (Shimabukuro et al., 1998).

$\mathrm{Na}$ análise comparativa entre os índices NDVI e EVI, utilizaram-se imagens compostas de 16 dias (Huete et al., 1999) do sensor Modis à bordo

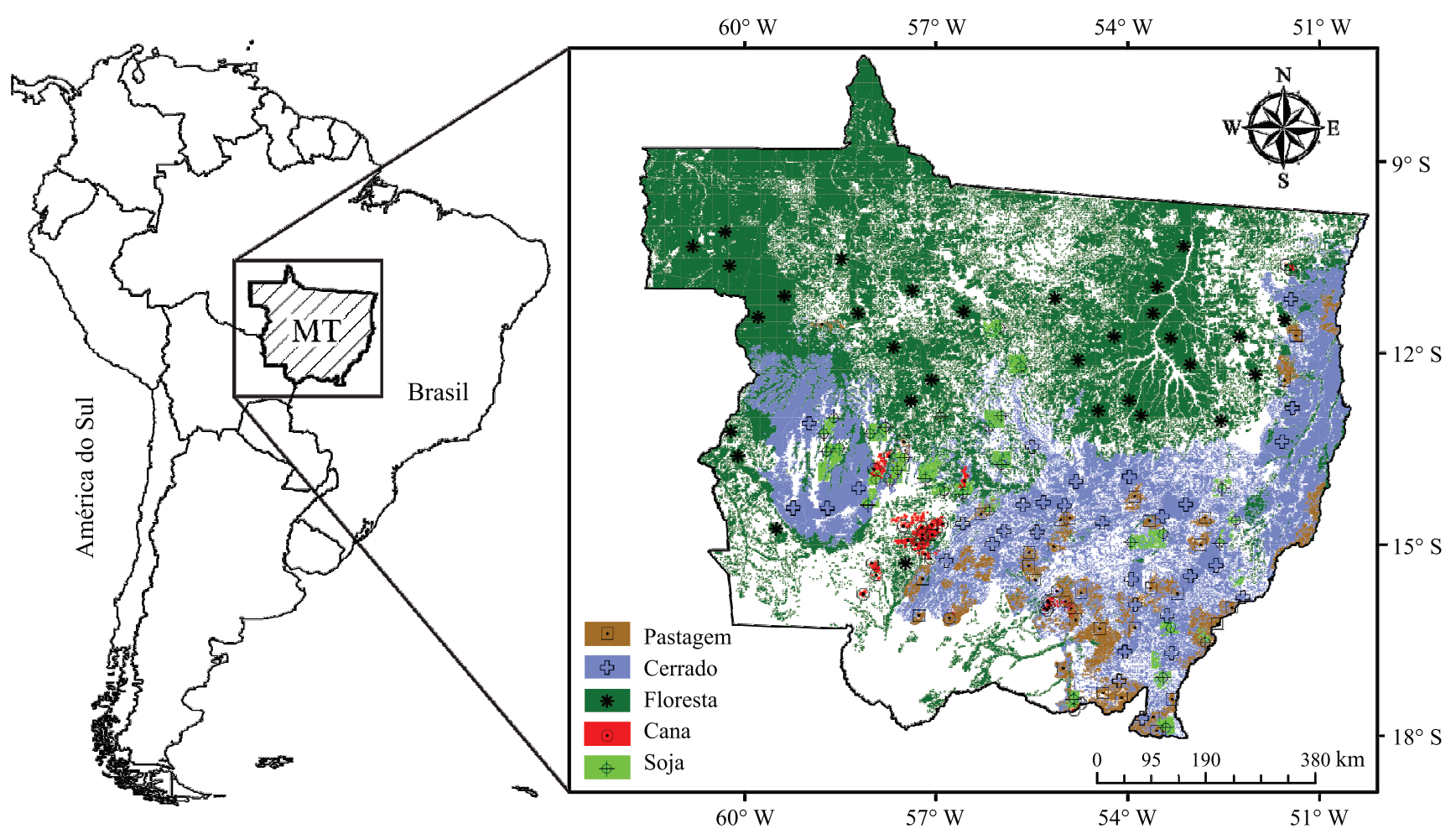

Figura 1. Alocação dos pontos amostrais sobre as classes soja, cana-de-açúcar, pastagem, cerrado e floresta, avaliadas no Estado do Mato Grosso (MT). 
do satélite Terra, mais especificamente o produto MOD13Q1, coleção 5.0, com resolução espacial de $250 \mathrm{~m}$. O NDVI foi obtido por meio da equação, NDVI $=(I V P-V) /(I V P+V)$, em que: IVP e V são os fatores de reflectância bidirecionais de superfície nas bandas do infravermelho próximo (banda 2) e do vermelho (banda 1), respectivamente.

O EVI foi proposto a partir da combinação de outros dois índices de vegetação: o índice de vegetação ajustado para o solo (Savi) (Huete, 1988) e o índice de vegetação resistente à atmosfera (Arvi) (Kaufman \& Tanre, 1992). $O$ EVI é calculado por meio da seguinte equação: $\mathrm{EVI}=\mathrm{G}\left[(\mathrm{IVP}-\mathrm{V}) /\left(\mathrm{IVP}+\mathrm{C}_{1} \times \mathrm{V}-\mathrm{C}_{2} \times \mathrm{A}+\mathrm{L}\right)\right], \mathrm{em}$ que IVP, $\mathrm{V}$ e $\mathrm{A}$ são os fatores de reflectância bidirecionais de superfície nas bandas do infravermelho próximo (banda 2), do vermelho (banda 1) e do azul (banda 3), respectivamente; $G$ é o fator de ganho (2,5); $\mathrm{C}_{1}$ é o coeficiente de correção dos efeitos atmosféricos para a banda do vermelho (6); $\mathrm{C}_{2}$ é o coeficiente de correção dos efeitos atmosféricos para a banda do azul $(7,5)$; e L é o fator de correção para a interferência do solo (1). Os coeficientes $\mathrm{C}_{1}$ e $\mathrm{C}_{2}$ podem ser alterados de acordo com as condições regionais, e os produtos disponibilizados pelo EOS utilizam as especificações supracitadas (Huete et al., 1999).

Para a avaliação independente dos períodos em que os alvos podem apresentar respostas espectrais distintas frente aos dois índices de vegetação, efetuouse a separação da série temporal das imagens Modis em entressafra e pleno desenvolvimento da cultura da soja (safra). Para tanto, adotou-se o seguinte critério: período de entressafra de 2005/2006, compreendido entre maio (dia do ano, DA, 145) e novembro de 2005 (DA 289), e período de safra de 2005/2006, compreendido entre dezembro de 2005 (DA 337) e março de 2006 (DA 49). O número que acompanha a sigla DA refere-se ao primeiro dia do ano utilizado na composição de 16 dias do Modis pelo EOS. A escolha desses intervalos temporais foi feita com base no calendário agrícola da soja no Estado do Mato Grosso, em que o início da estação seca coincide com o início da entressafra, a partir do final de março, e o fim da estação seca coincide com o início do período de semeadura, entre outubro e novembro.

Cada uma das amostras selecionadas, representativa das classes estudadas, foi associada às imagens Modis, para extrair o valor correspondente dos índices de vegetação em cada uma. Em seguida, aplicou-se a análise de variância entre as classes e o teste de
Tukey-Kramer, a 5\% de probabilidade, para identificar diferenças espectrais significativas entre a soja e as demais classes, em cada imagem, para cada índice de vegetação. Nos casos de diferenças significativas para ambos os índices de vegetação, aplicou-se o teste de Wilcoxon pareado, também a $5 \%$ de probabilidade, para identificar diferenças que justifiquem a opção por um ou outro índice de vegetação na imagem do período em questão.

\section{Resultados e Discussão}

Para exemplificar o comportamento típico de cada classe frente aos índices de vegetação em cada período, foram escolhidas duas imagens representativas da entressafra (DA 209 e 225) e duas da safra (DA 1 e 17), posicionadas no centro de cada período. No período seco, considerou-se que a resposta espectral típica das áreas de soja foi uma mistura de resposta de palha (restos culturais) e de solo. Isso implicou em valores de índice de vegetação que se mantiveram abaixo de 0,2 , no EVI, e de 0,3 no NDVI(Figura 2), significativamente diferente das demais classes avaliadas em ambos os índices de vegetação, nessas datas (Galford et al., 2008; Arvor et al., 2011). A cultura da cana-de-açúcar também tende a apresentar queda nos valores do EVI ao longo do período de seca (Xavier et al., 2006). No entanto, a estação seca afetou de maneira mais pronunciada as áreas de pastagem e de cerrado, em que o estresse hídrico e a ocorrência de fogo causaram queda na biomassa verde (Klink \& Machado, 2005). Isto refletiu em baixos valores de índice de vegetação também para essas classes, próximos daqueles observados para a soja.

Os elevados valores de índice de vegetação, nas áreas de floresta, apontaram para uma condição de menor instabilidade em razão da seca. Isso pode ser atribuído à estrutura radicular desenvolvida dessas formações, em comparação à de pastagem (Nepstad et al., 1994), bem como à menor duração da estação seca nas áreas de floresta, que apresentam precipitação média anual geralmente superior às de cerrado natural (Klink \& Machado, 2005). Em alguns casos, as áreas de floresta podem apresentar aumentos no valor do EVI e do NDVI no período seco, em comparação ao período de chuvas, associado ao fenômeno conhecido como "green-up" (Huete et al., 2006). Esse fenômeno, classificado como uma anomalia que não condiz com o incremento de biomassa, pode estar ligado às variações 
no ângulo zenital solar ao longo do ano (Galvão et al., 2011). Assim, a classe floresta apresentou diferença expressiva nos valores de ambos os índices no período da entressafra, quando comparada às demais classes (Figura 2), o que deve ser explorado em métodos multitemporais de classificação de uso e cobertura do solo na região Amazônica.

Semelhantemente ao observado nas imagens do DA 209 e 225 (Figura 2), verificou-se que, em grande parte do período da entressafra, para ambos os índices de vegetação, a classe soja apresentou diferenças significativas em comparação às demais (Tabela 1). Para o EVI, a classe soja diferiu significativamente de todas as demais entre as imagens DA 177 a 225 e na DA 257, e, para o NDVI, entre as imagens DA 161 a 273. Portanto, ambos os índices de vegetação provaram ser eficazes para discriminar áreas de produção de soja das áreas de cana-de-açúcar, pastagem, cerrado e floresta, no período de entressafra da soja, com dados Modis. Nas imagens em que ambos foram eficazes, o NDVI foi significativamente superior ao EVI (Tabela 1). Dessa forma, no período da entressafra, o NDVI apresentou potencial de contraste significativamente superior ao do EVI para discriminar a classe soja das demais classes avaliadas.
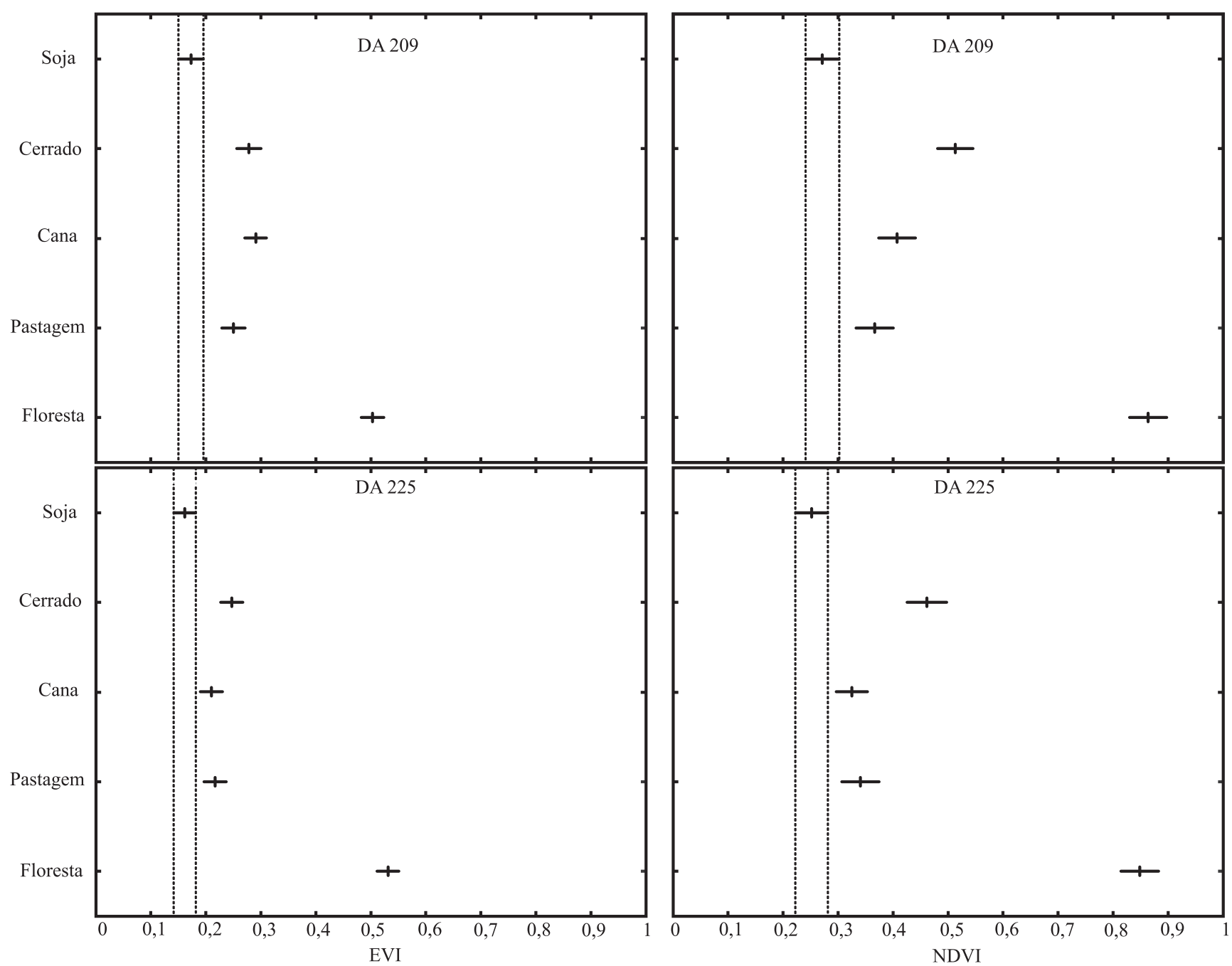

Figura 2. Análise comparativa da resposta espectral da classe soja frente à das classes cana-de-açúcar, pastagem, cerrado e floresta no EVI e no NDVI, para a imagem dia do ano (DA) 209 e 225 (período central da entressafra). O centro da barra representa a média, e as ramificações laterais o erro-padrão médio das 31 observações, pelo teste de Tukey-Kramer, a 5\% de probabilidade. 
O período DA 161 a 273 foi o mais propício para o aproveitamento do potencial do NDVI em classificações multitemporais, via séries temporais de índices de vegetação do sensor Modis, no Estado do Mato Grosso. Essa informação é de grande valia para os diversos métodos de classificação multitemporal de imagens, que utilizam, conjuntamente, informações espectrais dos períodos da entressafra e da safra, com o propósito de identificação e mapeamento da soja (Rizzi et al., 2009; Epiphanio et al., 2010; Arvor et al., 2011). A metodologia CEI (Rizzi et al., 2009), por exemplo, trabalha com a imagem de mínimo EVI e utiliza parte deste período (DA 241 a 305). Neste caso, o CEI pode ser aplicado, ao se substituir a imagem de mínimo EVI pela imagem de mínimo NDVI. Ressalta-se que, mesmo que o método CEI seja aplicado e mantenha-se apenas o EVI, o período utilizado para a geração da imagem de mínimo poderia ser antecipado, uma vez que, na imagem do DA 241, as classes cana-de-açúcar e soja não diferiram significativamente no EVI. A antecipação do período pode evitar a utilização de imagens contaminadas por nuvens e sombra de nuvens, típicas dessa época do ano no Estado do Mato Grosso. Observou-se que o período antecipado DA 177 a 241 coincide com as imagens de EVI do Modis que apresentaram maior potencial de contraste entre a soja e as demais classes, em ambos os índices de vegetação.

As imagens DA 1 e 17 coincidem com a época de chuvas na região e, consequentemente, com o período do cultivo da soja, evidenciado pelos altos valores de

Tabela 1. Índices de vegetação que apresentaram diferenças significativas na análise comparativa entre a classe soja e as classes cana-de-açúcar, pastagem, cerrado e floresta, no período da entressafra

\begin{tabular}{|c|c|c|c|c|}
\hline $\begin{array}{l}\text { Dia do } \\
\text { ano }\end{array}$ & $\begin{array}{c}\text { Soja vs. } \\
\text { cana }\end{array}$ & $\begin{array}{l}\text { Soja vs. } \\
\text { cerrado }\end{array}$ & $\begin{array}{c}\text { Soja vs. } \\
\text { pastagem }\end{array}$ & $\begin{array}{l}\text { Soja vs. } \\
\text { floresta }\end{array}$ \\
\hline 145 & ns & NDVI & ns & NDVI \\
\hline 161 & NDVI & NDVI*/EVI & NDVI & NDVI*/EVI \\
\hline 177 & NDVI*/EVI & NDVI*/EVI & NDVI*/EVI & NDVI*/EVI \\
\hline 193 & NDVI*/EVI & NDVI*/EVI & NDVI*/EVI & NDVI*/EVI \\
\hline 209 & NDVI*/EVI & NDVI*/EVI & NDVI*/EVI & NDVI*/EVI \\
\hline 225 & NDVI*/EVI & NDVI*/EVI & NDVI*/EVI & NDVI*/EVI \\
\hline 241 & NDVI & NDVI*/EVI & NDVI*/EVI & NDVI*/EVI \\
\hline 257 & NDVI*/EVI & NDVI*/EVI & NDVI*/EVI & NDVI*/EVI \\
\hline 273 & NDVI & NDVI & NDVI*/EVI & NDVI*/EVI \\
\hline 289 & NDVI*/EVI & ns & ns & NDVI*/EVI \\
\hline
\end{tabular}

${ }^{\text {ns }}$ Não significativo, na comparação entre a classe soja e as demais, para ambos os índices de vegetação, pelo teste de Tukey-Kramer, a 5\% de probabilidade. *Índice de vegetação significativo pelo teste de Wilcoxon pareado, a $5 \%$ de probabilidade. índice de vegetação para esta classe (Figura 3). Porém, a distribuição dos valores de índice de vegetação das imagens DA 1 e 17 , em torno de 0,7 , no EVI, e de 0,8 no NDVI, não equivalem ao pico da resposta das áreas de soja, pois este é temporalmente curto (menor que 30 dias) e alcançado em tempos diferentes, em razão, principalmente, da data de semeadura. Isso pode ser atribuído à maior variabilidade (erro-padrão médio) dos valores nos índices de vegetação, quando comparados com a entressafra. É esperado que, durante o pico de biomassa, uma lavoura de soja em bom estado de desenvolvimento e sem problemas fitossanitários graves supere o valor de 0,8 , no EVI, e de 0,85 no NDVI. Na época das chuvas, as demais classes também tendem a alcançar maior quantidade de biomassa verde na época das chuvas, o que pode ocasionar problemas de confusão espectral entre as classes, em decorrência da baixa sensibilidade destes índices em detectar diferenças sob condições de biomassa elevada (Huete et al., 2002).

De fato, constatou-se a inaptidão do NDVI em discriminar as classes soja e floresta durante todo o período de crescimento e desenvolvimento da soja no período de safra (Tabela 2). Entretanto, o EVI mostrou-se eficiente para discriminar a classe soja, tanto da floresta quanto do cerrado, entre as imagens DA 1 a 33. Também foi possível discriminar as classes cana-de-açúcar e pastagem da classe soja, por meio do EVI, durante os DA 353 a 17 e DA 353 a 33, respectivamente. No NDVI, novamente houve confusão espectral entre a soja e o cerrado, tendo-se observado diferenças significativas entre essas classes apenas na imagem DA 17. Contudo, frente às classes cana-de-açúcar e pastagem, o NDVI foi eficaz na discriminação da classe soja apenas no período central da safra, nas imagens DA 1 e 17. Mesmo nessas imagens e classes, em que ambos os índices de vegetação foram eficazes, o EVI mostrou-se significativamente superior, com potencial de contraste para a discriminação de áreas de soja, em comparação ao NDVI.

Wardlow et al. (2007), ao explorar imagens de índices de vegetação do Modis nas Grandes Planícies Centrais dos Estados Unidos, não identificaram diferenças significativas entre o EVI e o NDVI para discriminar culturas agrícolas, dentre elas a soja. Posteriormente, Wardlow \& Egbert (2008) aplicaram um método de classificação hierárquica sobre os dados de NDVI, para classificar as diferentes culturas agrícolas sob 
diferentes condições de manejo. Os resultados obtidos por esses autores indicaram exatidão global superior a $80 \%$ para o método de classificação testado. Chang et al. (2007) também avaliaram o desempenho das séries temporais de dados do sensor Modis, de bandas 1-7 e de NDVI, em resolução espacial de $500 \mathrm{~m}$, para mapear milho e soja nos Estados Unidos, por meio de abordagem baseada em árvores de regressão sobre métricas extraídas das séries temporais dos dados Modis. Esses autores observaram boa concordância com os dados oficiais do National Agricultural Statistics Service, com ligeira variação para a soja e para o milho de 4 e $6 \%$, respectivamente.
Porém, cabe destacar que, nas áreas avaliadas, não há ocorrência de florestas tropicais, como no Estado do Mato Grosso, onde a saturação assintótica do NDVI fica evidente, tanto sobre a floresta quanto sobre as áreas de soja em pleno desenvolvimento, o que representa problema de confusão espectral entre estas classes no período das chuvas. Portanto, o desenvolvimento de métodos mais eficientes para a classificação de áreas agrícolas, que possam oferecer subsídios para modelos de previsão e estimativa de safras no Brasil, deve levar em consideração especificidades regionais.

Semelhantemente ao observado na entressafra, os resultados permitiram definir o período DA 353 a
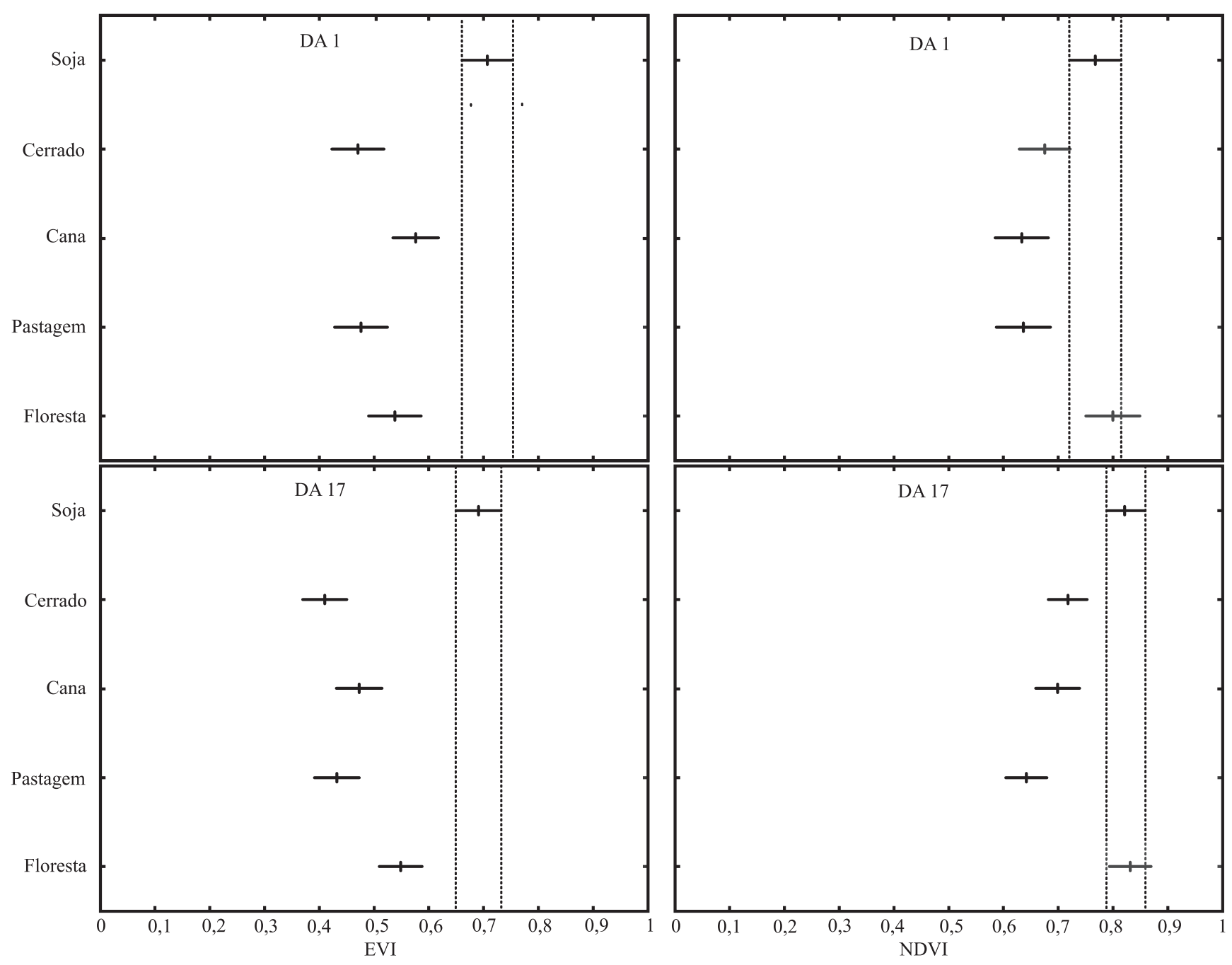

Figura 3. Análise comparativa da resposta espectral da classe soja frente à das classes cana-de-açúcar, pastagem, cerrado e floresta no EVI e no NDVI, para as imagens dia do ano (DA) 1 e 17 (período central da safra). O centro da barra representa a média, e as ramificações laterais o erro-padrão médio das 31 observações, pelo teste de Tukey-Kramer, a 5\% de probabilidade. 
33 como o mais propício para a seleção de imagens Modis, para aproveitar o melhor potencial de contraste do EVI, com vistas à discriminação da classe soja frente às demais classes, exceto a floresta (Tabela 2).

Alguns métodos de classificação multitemporal de imagens de sensoriamento remoto utilizam apenas o período da safra para a extração de informações que permitam discriminar culturas agrícolas (Wardlow et al., 2007). Assim, independentemente de se utilizar ou não informações da entressafra, recomenda-se o período DA 353 a 33, tanto para a aplicação do método de classificação multitemporal no Estado do Mato Grosso, quanto para o aproveitamento do maior contraste oferecido pelo EVI, na safra. Contudo, a utilização de um período estendido, em relação a essas datas, com base nos indícios de melhor potencial de separabilidade espectral do EVI, também é plausível. Isso porque o melhor período para separar a área de soja das demais é durante o pico de biomassa fotossinteticamente ativa, que pode apresentar janela temporal mais flexível em razão da ocorrência de semeadura precoce ou tardia da soja, o que aumenta as chances de obtenção de dados sem a contaminação por nuvens ou sombra de nuvens. Porém, no Mato Grosso, particularmente nas regiões de produção de algodão, essa flexibilidade não deve ir além de meados de março (DA 49), em decorrência da possibilidade de confusão espectral com essa cultura, que apresenta resposta espectral próxima à da soja durante o pico de biomassa. Portanto, fica evidente a importância do conhecimento do calendário agrícola na região, ao se empregar métodos multitemporais com vistas a classificar culturas agrícolas.

Tabela 2. Índices de vegetação que apresentaram diferenças significativas na análise comparativa entre a classe soja e as classes cana-de-açúcar, pastagem, cerrado e floresta, para o período da safra.

\begin{tabular}{lcccc}
\hline $\begin{array}{l}\text { Dia do } \\
\text { ano }\end{array}$ & $\begin{array}{c}\text { Soja vs. } \\
\text { cana }\end{array}$ & $\begin{array}{c}\text { Soja vs. } \\
\text { cerrado }\end{array}$ & $\begin{array}{c}\text { Soja vs. } \\
\text { pastagem }\end{array}$ & Soja vs. floresta \\
\hline 337 & ns & ns & ns & ns \\
353 & EVI & EVI & EVI & ns \\
1 & EVI*/NDVI & EVI & EVI*/NDVI & EVI \\
17 & EVI*/NDVI & EVI*/NDVI & EVI*/NDVI & EVI \\
33 & ns & EVI & EVI & EVI \\
49 & ns & ns & ns & ns \\
\hline
\end{tabular}

${ }^{\text {ns }}$ ão significativo, na comparação entre a classe soja e as demais classes, para ambos os índices de vegetação, pelo teste de Tukey-Kramer, a 5\% de probabilidade. *Índice de vegetação significativo pelo teste de Wilcoxon pareado, a $5 \%$ de probabilidade.
Como o NDVI foi mais eficiente que o EVI na discriminação da classe soja em relação às demais classes testadas na entressafra, e o EVI foi mais indicado na safra, sobretudo em regiões de floresta e cerrado, espera-se que o desenvolvimento de métodos de análises multitemporais que utilizem de forma combinada o NDVI, na entressafra, e o EVI, na safra, apresentem bons resultados. Além disso, mesmo que as classes testadas sejam representativas da região estudada, recomenda-se estender a análise frente a outros alvos, principalmente aqueles que possam apresentar algum tipo de confusão espectral com áreas cultivadas com soja, particularmente no verão, que é o pico da safra agrícola na região.

\section{Conclusões}

1. A classificação de áreas de soja no Mato Grosso, por meio de métodos multitemporais baseados nas séries de índice de vegetação do Modis, apresenta melhores resultados com a utilização combinada do índice de vegetação da diferença normalizada (NDVI), no período da entressafra, e do índice de vegetação realçado (EVI) no período da safra.

2. O NDVI apresenta melhor desempenho para discriminação de áreas de produção de soja no Estado do Mato Grosso, no período da entressafra, quando comparado ao EVI.

3. No período da safra, o melhor desempenho para a discriminação de áreas cultivadas com soja é atribuído ao EVI, e o NDVI é inapto para discriminar áreas de soja e de floresta durante esse período no Estado do Mato Grosso.

4. Os melhores períodos para a discriminação espectral das áreas de produção de soja em relação às demais classes, referem-se às imagens Modis dos dias do ano 161 a 273 e 353 a 33, para entressafra e safra, respectivamente.

\section{Referências}

ARVOR, D.; JONATHAN, M.; MEIRELLES, M.S.P.; DUBREUIL, V.; DURIEUX, L. Classification of MODIS EVI time series for crop mapping in the state of Mato Grosso, Brazil. International Journal of Remote Sensing, v.32, p.7847-7871, 2011.

BRASIL. Ministério do Meio Ambiente. Mapeamento de cobertura vegetal do bioma Cerrado: relatório final. Brasília: MMA, 2007. 93p. 
BRASIL. Ministério do Meio Ambiente. Relatório técnico de monitoramento do desmatamento no bioma Cerrado, 2002 a 2008: dados revisados. Brasília: MMA, 2009. 67p.

CHANG, J.; HANSEN, M.C.; PITTMAN, K.; CARROLL, M.; DIMICELI, C. Corn and soybean mapping in the United States using MODN time-series data sets. Agronomy Journal, v.99, p.1654-1664, 2007.

COMPANHIA NACIONAL DE ABASTECIMENTO. Séries históricas relativas às safras 1976/77 a 2011/2012 de área plantada, produtividade e produção. Disponível em: $<$ http:// www.conab.gov.br/conteudos.php? $\mathrm{a}=1252 \& \mathrm{t}=>$. Acesso em: 10 jul. 2012.

DIDAN, K.; HUETE, A. MODIS vegetation index product series: collection 5 change summary. Tucson: The University of Arizona, 2006. 17p. Available at: <http://landweb.nascom.nasa.gov/QA WWW/forPage/MOD13_VI_C5_Changes_Document_06_28_06. pdf $>$. Accessed on: 16 July 2012.

EPIPHANIO, R.D.V.; FORMAGGIO, A.R.; RUDORFF, B.F.T.; MAEDA, E.E.; LUIZ, A.J.B. Estimating soybean crop areas using spectral-temporal surfaces derived from MODIS images in Mato Grosso, Brazil. Pesquisa Agropecuária Brasileira, v.45, p.72-80, 2010.

FREITAS, R.M. de; ARAI, E.; ADAMI, M.; FERREIRA, A.S.; SATO, F.Y.; SHIMABUKURO, Y.E.; ROSA, R.R.; ANDERSON, L.O.; RUDORFF, B.F.T. Virtual laboratory of remote sensing time series: visualization of MODIS EVI2 data set over South America. Journal of Computational Interdisciplinary Sciences, v.2, p.57-68, 2011.

FRIEDL, M.A.; MCIVER, D.K.; HODGES, J.C.F.; ZHANG, X.Y.; MUCHONEY, D.; STRAHLER, A.H.; WOODCOCK, C.E.; GOPAL, S.; SCHNEIDER, A.; COOPER, A.; BACCINI, A.; GAO, F.; SCHAAF, C. Global land cover mapping from MODIS: algorithms and early results. Remote Sensing of Environment, v.83, p.287-302, 2002.

GALFORD, G.L.; MUSTARD, J.F.; MELILLO, J.; GENDRIN, A.; CERRI, C.C.; CERRI, C.E.P. Wavelet analysis of MODIS time series to detect expansion and intensification of row-crop agriculture in Brazil. Remote Sensing of Environment, v.112, p.576-587, 2008.

GALVÃO, L.S.; SANTOS, J.R. dos; ROBERTS, D.A.; BREUNIG, F.M.; TOOMEY, M.; MOURA, Y.M. de. On intra-annual EVI variability in the dry season of tropical forest: a case study with MODIS and hyperspectral data. Remote Sensing of Environment, v.115, p.2350-2359, 2011.

GITELSON, A.A.; KAUFMAN, Y.J.; MERZLYAK, M.N. Use of a green channel in remote sensing of global vegetation from EOS-MODIS. Remote Sensing of Environment, v.58, p.289-298, 1996.

HUETE, A.; DIDAN, K.; MIURA, T.; RODRIGUEZ, E.P.; GAO, X.; FERREIRA, L.G. Overview of the radiometric and biophysical performance of the MODIS vegetation indices. Remote Sensing of Environment, v.83, p.195-213, 2002.

HUETE, A.; JUSTICE, C.; LEEUWEN, W. van. MODIS vegetation index (MOD 13): algorithm theoretical basis document. Version
3. Washington: National Aeronautics and Space Administration, 1999. 129p.

HUETE, A.R. A soil-adjusted vegetation index (SAVI). Remote Sensing of Environment, v.25, p.295-309, 1988.

HUETE, A.R.; DIDAN, K.; SHIMABUKURO, Y.E.; RATANA, P.; SALESKA, S.R.; HUTYRA, L.R.; YANG, W.; NEMANI, R.R.; MYNENI, R. Amazon rainforests green-up with sunlight in dry season. Geophysical Research Letters, v.33, p.1-4, 2006. Doi: 10.1029/2005GL025583.

HUETE, A.R.; LIU, H.Q.; BATCHILY, K.; LEEUWEN, W. van. A comparison of vegetation indices over a global set of TM images for EOS-MODIS. Remote Sensing of Environment, v.59, p.440-451, 1997.

JUSTICE, C.O.; TOWNSHEND, J.R.G.; VERMOTE, E.F.; MASUOKA, E.; WOLFE, R.E.; SALEOUS, N.; ROY, D.P.; MORISETTE, J.T. An overview of MODIS Land data processing and product status. Remote Sensing of Environment, v.83, p.3-15, 2002.

JUSTICE, C.O.; VERMOTE, E.; TOWNSHEND, J.R.G.; DEFRIES, R.; ROY, D.P.; HALL, D.K.; SALOMONSON, V.V.; PRIVETTE, J.L.; RIGGS, G.; STRAHLER, A.; LUCHT, W.; MYNENI, R.B.; KNYAZIKHIN, Y.; RUNNING, S.W.; NEMANI, R.R.; ZHENGMING, W.; HUETE, A.R.; VAN LEEUWEN, W.; WOLFE, R.E.; GIGLIO, L.; MULLER, J.; LEWIS, P.; BARNSLEY, M.J. The Moderate Resolution Imaging Spectroradiometer (MODIS): land remote sensing for global change research. IEEE Transactions on Geoscience and Remote Sensing, v.36, p.1228-1249, 1998.

KAUFMAN, Y.J.; TANRE, D. Atmospherically resistant vegetation index (ARVI) for EOS-MODIS. IEEE Transactions on Geoscience and Remote Sensing, v.30, p.261-270, 1992.

KLINK, C.A.; MACHADO, R.B. Conservation of the Brazilian Cerrado. Conservation Biology, v.19, p.707-713, 2005.

NEPSTAD, D.C.; CARVALHO, C.R. de; DAVIDSON, E.A.; JIPP, P.H.; LEFEBVRE, P.A.; NEGREIROS, G.H.; SILVA, E.D. da; STONE, T.A.; TRUMBORE, S.E.; VIEIRA, S. The role of deep roots in the hydrological and carbon cycles of Amazonian forests and pastures. Nature, v.372, p.666-669, 1994.

RIZZI, R.; RISSO, J.; EPIPHANIO, R.D.V.; RUDORFF, B.F.T.; FORMAGGIO, A.R.; SHIMABUKURO, Y.E.; FERNANDES, S.L. Estimativa da área de soja no Mato Grosso por meio de imagens MODIS. In: SIMPÓSIO BRASILEIRO DE SENSORIAMENTO REMOTO, 14., 2009. Natal. Anais. São José dos Campos: Instituto Nacional de Pesquisas Espaciais, 2009. p.387-394.

ROUSE, J.W.; HASS, R.H.; SCHELL, J.A.; DEERING, D.W. Monitoring vegetation systems in the Great Plains with ERTS. In: EARTH RESOURCES TECHNOLOGY SATELLITE SYMPOSIUM, 3., 1973, Washington. Proceedings. Washington: NASA, 1974. p.309-317.

RUDORFF, B.F.T.; ADAMI, M.; RISSO, J.; AGUIAR, D.A. de; PIRES, B.; AMARAL, D.; FABIANI, L.; CECARELLI, I. Remote sensing images to detect soy plantations in the amazon biome - the soy moratorium initiative. Sustainability, v.4, p.1074-1088, 2012. 
RUDORFF, B.F.T.; AGUIAR, D.A.; SILVA, W.F.; SUGAWARA, L.M.; ADAMI, M.; MOREIRA, M.A. Studies on the rapid expansion of sugarcane for ethanol production in São Paulo State (Brazil) using Landsat data. Remote Sensing, v.2, p.1057-1076, 2010.

SHIMABUKURO, Y.E.; BATISTA, G.T.; MELLO, E.M.K.; MOREIRA, J.C.; DUARTE, V. Using shade fraction image segmentation to evaluate deforestation in Landsat Thematic Mapper images of the Amazon Region. International Journal of Remote Sensing, v.19, p.535-541, 1998.

TOWNSHEND, J.R.G.; JUSTICE, C.O. Towards operational monitoring of terrestrial systems by moderate-resolution remote sensing. Remote Sensing of Environment, v.83, p.351-359, 2002.

VERMOTE, E.F.; EL SALEOUS, N.Z.; JUSTICE, C.O. Atmospheric correction of MODIS data in the visible to middle infrared: first results. Remote Sensing of Environment, v.83, p.97-111, 2002.

WARDLOW, B.D.; EGBERT, S.L. Large-area crop mapping using time-series MODIS $250 \mathrm{~m}$ NDVI data: an assessment for the U.S. Central Great Plains. Remote Sensing of Environment, v.112, p.1096-1116, 2008.
WARDLOW, B.D.; EGBERT, S.L.; KASTENS, J.H. Analysis of time-series MODIS $250 \mathrm{~m}$ vegetation index data for crop classification in the U.S. Central Great Plains. Remote Sensing of Environment, v.108, p.290-310, 2007.

WARDLOW, B.D.; KASTENS, J.H.; EGBERT, S.L. Using USDA crop progress data for the evaluation of greenup onset date calculated from MODIS 250-meter data. Photogrammetric Engineering and Remote Sensing, v.72, p.1225-1234, 2006.

WOLFE, R.E.; NISHIHAMA, M.; FLEIG, A.J.; KUYPER, J.A.; ROY, D.P.; STOREY, J.C.; PATT, F.S. Achieving sub-pixel geolocation accuracy in support of MODIS land science. Remote Sensing of Environment, v.83, p.31-49, 2002.

XAVIER, A.C.; RUDORFF, B.F.T.; SHIMABUKURO, Y.E.; BERKA, L.M.S.; MOREIRA, M.A. Multi-temporal analysis of MODIS data to classify sugarcane crop. International Journal of Remote Sensing, v.27, p.755-768, 2006.

ZHANG, X.Y.; FRIEDL, M.A.; SCHAAF, C.B.; STRAHLER, A.H.; HODGES, J.C.F.; GAO, F.; REED, B.C.; HUETE, A. Monitoring vegetation phenology using MODIS. Remote Sensing of Environment, v.84, p.471-475, 2003.

Recebido em 31 de julho de 2011 e aprovado em 31 de julho de 2012 